Kragujevac Journal of Mathematics

Volume 42(2) (2018), Pages 209-215.

\title{
NOTE ON THE RANDIĆ ENERGY OF GRAPHS
}

\author{
JUN HE ${ }^{1}$, YAN-MIN LIU ${ }^{1}$, AND JUN-KANG TIAN ${ }^{1}$
}

\begin{abstract}
If $G$ is a graph on $n$ vertices, and $d_{i}$ is the degree of its $i$-th vertex, then the Randic matrix of $G$ is the square matrix of order $n$ whose $(i, j)$-entry is equal to $1 / \sqrt{d_{i} d_{j}}$ if the $i$-th and $j$-th vertex of $G$ are adjacent, and zero otherwise. In this note, we obtain some new lower and upper bounds for the Randić energy.
\end{abstract}

\section{INTRODUCTION}

Let $G$ be a simple connected graph with vertex set $V(G)=\left\{v_{1}, v_{2}, \ldots, v_{n}\right\}$ and edge set $E(G)$. Let $d_{i}$ denote the degree of vertex $v_{i}$, where $i=1,2, \ldots, n$. The maximum vertex degree is denoted by $\Delta$. Use the notation $v_{i} \sim v_{j}$, if two vertices $v_{i}$ and $v_{j}$ of $G$ are adjacent.

The Randić matrix $R=R(G)$ of $G$ is defined as

$$
r_{i j}= \begin{cases}\frac{1}{\sqrt{d_{i} d_{j}}}, & \text { if the vertices } v_{i} \text { and } v_{j} \text { are adjacent, } \\ 0, & \text { if the vertices } v_{i} \text { and } v_{j} \text { are not adjacent, } \\ 0, & \text { if } i=j\end{cases}
$$

The Randic matrix is real symmetric, so we can order the eigenvalues of its Randic matrix so that $\rho_{1} \geq \rho_{2} \geq \ldots \geq \rho_{n}$. The Randić energy of the graph $G$ is defined in $[2,3,6,7]$ as:

$$
R E=R E(G)=\sum_{i=1}^{n}\left|\rho_{i}\right|
$$

As usual, the adjacency matrix $A=A(G)$ of the graph $G$ is defined so that its $(i, j)$-element is equal to unity if the vertices $v_{i}$ and $v_{j}$ are adjacent, and is equal to

Key words and phrases. Randić energy, Randić matrix, bounds.

2010 Mathematics Subject Classification. Primary: 05C50. Secondary: 15A18.

Received: May 10, 2016.

Accepted: February 02, 2017. 
zero otherwise. Let $L=D-A$ and $Q=D+A$ be, respectively, the Laplacian and the signless Laplacian matrix of the graph $G[9,10,12]$. If we define

$$
\mathcal{L}=D^{-1 / 2} L D^{-1 / 2} \quad \text { and } \quad \mathcal{Q}=D^{-1 / 2} Q D^{-1 / 2}
$$

where $D$ is the diagonal matrix of vertex degrees, are the normalized Laplacian and normalized signless Laplacian matrix. Then, evidently,

$$
\mathcal{L}=I_{n}-R \quad \text { and } \quad \mathcal{Q}=I_{n}+R
$$

The paper is organized as follows. In Section 2, we give a list of some previously known results. In Section 3, we present some lower and upper bounds on the Randić energy.

\section{Preliminary Lemmas}

In order to obtain bounds for the Randić energy of $G$, we need some lemmas.

Lemma 2.1. [1] Let $G$ be a graph with $n$ vertices and Randić matrix $R$. Then

$$
\begin{gathered}
\operatorname{tr}(R)=0, \quad \operatorname{tr}\left(R^{2}\right)=2 \sum_{i \sim j} \frac{1}{d_{i} d_{j}} \\
\operatorname{tr}\left(R^{4}\right)=\sum_{i=1}^{n}\left(\sum_{i \sim j} \frac{1}{d_{i} d_{j}}\right)^{2}+\sum_{i \neq j} \frac{1}{d_{i} d_{j}}\left(\sum_{k \sim i, k \sim j} \frac{1}{d_{k}}\right)^{2} .
\end{gathered}
$$

Lemma 2.2. [1] A simple connected graph $G$ has two distinct Randic eigenvalues if and only if $G$ is complete.

Lemma 2.3. [13] Let $a_{1}, a_{2}, \ldots, a_{n}$ be non-negative numbers. Then

$$
\begin{aligned}
n\left[\frac{1}{n} \sum_{j=1}^{n} a_{j}-\left(\prod_{j=1}^{n} a_{j}\right)^{1 / n}\right] & \leq n \sum_{j=1}^{n} a_{j}-\left(\sum_{j=1}^{n} \sqrt{a_{j}}\right)^{2} \\
& \leq n(n-1)\left[\frac{1}{n} \sum_{j=1}^{n} a_{j}-\left(\prod_{j=1}^{n} a_{j}\right)^{1 / n}\right] .
\end{aligned}
$$

Lemma 2.4. [11] Let $G$ be a graph on $n$ vertices which has at least one edge, $v_{1} \geq$ $\ldots \geq v_{n}$ be the eigenvalues of the Laplacian L. Then

$$
v_{1} \geq \Delta+1
$$

Moreover, if $G$ is connected, then the equality holds if and only if $\Delta=n-1$. 


\section{Main Results}

It has been proved that $\rho_{1}=1$ is the largest $R$-eigenvalues with the Perron-Frobenius vector $\alpha^{T}=\left(\sqrt{d_{1}}, \ldots, \sqrt{d_{n}}\right)($ see $[4,6,8])$.

Theorem 3.1. Let $G$ be a graph with $n$ vertices and at least one edge. Then

$$
R E(G) \geq 1+\left(2 \sum_{i \sim j} \frac{1}{d_{i} d_{j}}-1\right) \sqrt{\frac{2 \sum_{i \sim j} \frac{1}{d_{i} d_{j}}-1}{\sum_{i=1}^{n}\left(\sum_{i \sim j} \frac{1}{d_{i} d_{j}}\right)^{2}+\sum_{i \neq j} \frac{1}{d_{i} d_{j}}\left(\sum_{k \sim i, k \sim j} \frac{1}{d_{k}}\right)^{2}-1} .}
$$

Proof. Using Hölder inequality, we have

$$
\sum_{i=2}^{n} a_{i} b_{i} \leq\left(\sum_{i=2}^{n} a_{i}^{p}\right)^{1 / p}\left(\sum_{i=2}^{n} b_{i}^{q}\right)^{1 / q}
$$

which holds for any non-negative real numbers $a_{i}, b_{i}, i=2,3, \ldots, n$. Setting $a_{i}=|\rho|^{2 / 3}$, $b_{i}=|\rho|^{4 / 3}, p=3 / 2$ and $q=3$, we obtain

$$
\sum_{i=2}^{n}\left|\rho_{i}\right|^{2}=\sum_{i=2}^{n}\left|\rho_{i}\right|^{2 / 3} \sum_{i=2}^{n}\left|\rho_{i}\right|^{4 / 3} \leq\left(\sum_{i=2}^{n}\left|\rho_{i}\right|\right)^{2 / 3}\left(\sum_{i=2}^{n}\left|\rho_{i}\right|^{4}\right)^{1 / 3}
$$

that is

$$
R E(G)-1=\sum_{i=2}^{n}\left|\rho_{i}\right| \geq \sqrt{\frac{\left(\sum_{i=2}^{n}\left|\rho_{i}\right|^{2}\right)^{3}}{\sum_{i=2}^{n}\left|\rho_{i}\right|^{4}}}=\sqrt{\frac{\left(\operatorname{tr}\left(R^{2}\right)-1\right)^{3}}{\operatorname{tr}\left(R^{4}\right)-1}} .
$$

Hence we get the result.

We next derive a lower bound of the Randic energy in terms of the order $n$ and $\operatorname{det}(R)$.

Theorem 3.2. Let $G$ be a graph with $n$ vertices. Then

$$
R E(G) \geq 1+(n-1)|\operatorname{det}(R)|^{\frac{1}{n-1}},
$$

and the equality holds in (3.1) if and only if $G$ is a complete graph or a non-bipartite connected graph with three distinct Randić eigenvalues

$$
\left(1, \sqrt{\frac{2 \sum_{i \sim j} \frac{1}{d_{i} d_{j}}}{n-1}},-\sqrt{\frac{2 \sum_{i \sim j} \frac{1}{d_{i} d_{j}}}{n-1}}\right) .
$$

Proof. Using the arithmetic-geometric mean inequality, we obtain that

$$
R E(G)=\rho_{1}+\sum_{i=2}^{n}\left|\rho_{i}\right| \geq 1+(n-1)\left(\prod_{i=2}^{n}\left|\rho_{i}\right|\right)^{\frac{1}{n-1}}=1+(n-1)|\operatorname{det}(R)|^{\frac{1}{n-1}},
$$


and equality holds if and only if $\rho_{1}=1$ and $\left|\rho_{2}\right|=\ldots=\left|\rho_{n}\right|=\sqrt{\frac{2 \sum_{i \sim j} \frac{1}{d_{i} d_{j}}}{n-1}}$, which is discussed in the proof of Theorem 4 in [4].

Now the proof is complete.

Theorem 3.3. Let $G$ be a graph with $n$ vertices. Then

$$
R E(G) \geq 1+\sqrt{2 \sum_{i \sim j} \frac{1}{d_{i} d_{j}}-1+(n-1)(n-2)(\operatorname{det}(R))^{\frac{2}{n-1}}},
$$

and

$$
R E(G) \leq 1+\sqrt{(n-2)\left(2 \sum_{i \sim j} \frac{1}{d_{i} d_{j}}-1\right)+(n-1)(\operatorname{det}(R))^{\frac{2}{n-1}}} .
$$

Proof. Let $a_{i}=\rho_{i}^{2}, i=2, \ldots, n$. Then by Lemma 2.1 and Lemma 2.3 we obtain

$$
K \leq(n-1) \sum_{i=2}^{n} \rho_{i}^{2}-\left(\sum_{i=2}^{n}\left|\rho_{i}\right|\right)^{2} \leq(n-2) K,
$$

that is,

$$
K \leq(n-1)\left(2 \sum_{i \sim j} \frac{1}{d_{i} d_{j}}-1\right)-(R E(G)-1)^{2} \leq(n-2) K,
$$

where

$$
\begin{aligned}
K & =(n-1)\left[\frac{1}{n-1} \sum_{i=2}^{n} \rho_{i}^{2}-\left(\prod_{i=2}^{n} \rho_{i}^{2}\right)^{\frac{1}{n-1}}\right] \\
& =(n-1)\left[\frac{1}{n-1}\left(2 \sum_{i \sim j} \frac{1}{d_{i} d_{j}}-1\right)-\left(\prod_{i=2}^{n}\left|\rho_{i}\right|\right)^{\frac{2}{n-1}}\right] \\
& =2 \sum_{i \sim j} \frac{1}{d_{i} d_{j}}-1-(n-1)\left(\operatorname{det}(R)^{\frac{2}{n-1}}\right.
\end{aligned}
$$

Hence we get the result.

Remark 3.1. Using the relation between the arithmetic and geometric means,

$$
(\operatorname{det}(R))^{2} \leq\left(\frac{2 \sum_{i \sim j} \frac{1}{d_{i} d_{j}}-1}{n-1}\right)^{n-1},
$$

and bearing in mind the upper bound in (3.2), we arrive at

$$
R E(G) \leq 1+\sqrt{(n-1)\left(2 \sum_{i \sim j} \frac{1}{d_{i} d_{j}}-1\right)}
$$

which is same as the result in $[4,8]$. 
Lemma 3.1. Let $G$ be a graph with $n$ vertices. Then

$$
\left|\rho_{n}\right| \geq \frac{1}{\Delta}
$$

Proof. Let $u_{1} \geq \ldots \geq u_{n}$ be the eigenvalues of the normalized Laplacian $\mathcal{L}$, by equality (1.1), we have

$$
\rho_{n}=1-u_{1} .
$$

Let $\|A\|$ be the spectral norm of a matrix $A$, by $\mathcal{L}=D^{-1 / 2} L D^{-1 / 2}$ and Lemma 2.4, we can get

therefore,

$$
\Delta+1 \leq\|L\| \leq\|D\|\|\mathcal{L}\|=\Delta u_{1}
$$

Then, by equality (3.3), we obtain

$$
u_{1} \geq 1+\frac{1}{\Delta}
$$

$$
\left|\rho_{n}\right| \geq \frac{1}{\Delta}
$$

Now the proof is complete.

Theorem 3.4. Let $G$ be a graph with $n \geq 2$ vertices. Then

$$
R E(G) \leq 1+\sqrt{\frac{2 \sum_{i \sim j} \frac{1}{d_{i} d_{j}}-1}{n-1}}+\sqrt{(n-2)\left(2 \sum_{i \sim j} \frac{1}{d_{i} d_{j}}-1-\frac{2 \sum_{i \sim j} \frac{1}{d_{i} d_{j}}-1}{n-1}\right)} .
$$

More, if $\frac{1}{\Delta} \geq \sqrt{\frac{2 \sum_{i \sim j} \frac{1}{d_{i} d_{j}}-1}{n-1}}$, then

$$
R E(G) \leq 1+\frac{1}{\Delta}+\sqrt{(n-2)\left(2 \sum_{i \sim j} \frac{1}{d_{i} d_{j}}-1-\frac{1}{\Delta^{2}}\right)}
$$

Proof. Using Cauchy-Schwarz inequality, we obtain

$$
\begin{aligned}
R E(G) & =\rho_{1}+\sum_{i=2}^{n-1}\left|\rho_{i}\right|+\left|\rho_{n}\right| \leq 1+\left|\rho_{n}\right|+\sqrt{(n-2) \sum_{i=2}^{n-1} \rho_{i}^{2}} \\
& \leq 1+\left|\rho_{n}\right|+\sqrt{(n-2)\left(2 \sum_{i \sim j} \frac{1}{d_{i} d_{j}}-1-\rho_{n}^{2}\right)} .
\end{aligned}
$$

Define a function $f(x)=1+x+\sqrt{(n-2)\left(2 \sum_{i \sim j} \frac{1}{d_{i} d_{j}}-1-x^{2}\right)}$, it is easy to see that the function $f(x)$ is monotonously decreasing in $x \geq \sqrt{\frac{2 \sum_{i \sim j} \frac{1}{d_{i} d_{j}}-1}{n-1}}$, which implies that the inequality (3.4) holds. 
By Lemma 3.1, if we have $\left|\rho_{n}\right| \geq \frac{1}{\Delta} \geq \sqrt{\frac{2 \sum_{i \sim j} \frac{1}{d_{i} d_{j}}-1}{n-1}}$, then

$$
f\left(\left|\rho_{n}\right|\right) \leq f\left(\frac{1}{\Delta}\right)
$$

which implies that the inequality (3.5) holds. Now the proof is complete.

Acknowledgements. He is supported by Science and technology Foundation of Guizhou province (Qian ke he Ji Chu [2016]1161); Guizhou province natural science foundation in China (Qian Jiao He KY [2016]255); The doctoral scientific research foundation of Zunyi Normal College (BS [2015]09). Liu is supported by National Natural Science Foundations of China (71461027); Science and technology talent training object of Guizhou province outstanding youth (Qian ke he ren zi [2015]06); Guizhou province natural science foundation in China (Qian Jiao He KY [2014]295); 2013, 2014 and 2015 Zunyi 15851 talents elite project funding; Zhunyi innovative talent team (Zunyi KH [2015]38). Tian is supported by the Science and Technology fund Project of GZ (Qian ke he J Zi [2015]2147, Qian jiao he KY [2015]451).

\section{REFERENCES}

[1] Ş. B. Bozkurt, A. D. Güngör, I. Gutman and A. S. Çevik, Randić matrix and Randić energy, MATCH Commun. Math. Comput. Chem. 64 (2010), 239-250.

[2] Ş. B. Bozkurt, A. D. Güngör and I. Gutman, Randić spectral radius and Randić energy, MATCH Commun. Math. Comput. Chem. 64 (2010), 321-334.

[3] Ş. B. Bozkurt and D. Bozkurt, Randić energy and Randić Estrada index of a graph, Eur. J. Pure Appl. Math. 5 (2012), 88-96.

[4] Ş. B. Bozkurt and D. Bozkurt, Sharp upper bounds for energy and randić energy, MATCH Commun. Math. Comput. Chem. 70 (2013), 669-680.

[5] I. Gutman, Bounds for total $\pi$-electron energy, Chem. Phys. Lett. 24 (1974), 283-285.

[6] I. Gutman, B. Furtula and Ş. B. Bozkurt, On Randić Energy, Linear Algebra Appl. 442 (2014), $50-57$.

[7] X. Li and Y. Shi, A survey on the Randić index, MATCH Commun. Math. Comput. Chem. 59 (2008), 127-156.

[8] B. Liu, Y. Huang and J. Feng, A note on the Randić spectral radius, MATCH Commun. Math. Comput. Chem. 68 (2012), 913-916.

[9] J. H. Koolen, V. Moulton and I. Gutman, Improving the McClelland inequality for total $\pi$-electron energy, Chem. Phys. Lett. 320 (2000), 213-216.

[10] J. H. Koolen and V. Moulton, Maximal energy graphs, Adv. Appl. Math. 26 (2001), 47-52.

[11] R. Merris, Laplacian matrices of graphs: a survey, Linear Algebra Appl. 197-198 (1994), 143-176.

[12] B. Zhou, Energy of a graph, MATCH Commun. Math. Comput. Chem. 51 (2004), 111-118.

[13] B. Zhou, I. Gutman and T. Aleksić, A note on the Laplacian energy of graphs, MATCH Commun. Math. Comput. Chem. 60 (2008), 441-446. 
${ }^{1}$ SCHOOL OF MATHEMATICS,

Zunyi Normal College,

Zunyi, Guizhou, 563002, P.R. China

E-mail address: hejunfan1@163.com

E-mail address: 546692653@qq.com

E-mail address: $17261717 @ q q . c o m$ 